Bereich der sog. Kernaufgaben, solange dieser Staat jedenfalls ein harter Staat bleibt, ja diese Eigenschaft sogar noch stärker ausbildet. Wer Opfer eines Lauschangriffs wird oder gegen Castor-Transporte demonstriert, wird diesen Staat denn auch kaum als »schlank « empfinden.

Auf der anderen Seite bewirken Maßnahmen zur Privatisierung der Sicherheitsgewährung Prozesse weiterer sozialer Separierung: Die Angebote privater "Sicherheits «-unternehmen kann schließlich nur diejenige annehmen, die über die notwendigen finanziellen Mittel verfügt ${ }^{46}$. Auch im Bereich ehemals staatlich erbrachter Dienstleistungen wie bei der Telekommunikation setzen sich die »Mechanismen des freien Marktes“ durch, wie ein Befürworter freimütig einräumt. »Das Spiel von Angebot und Nachfrage sowie die Ziele der Kostenminimierung und Gewinnmaximierung ersetzen dabei die bisher bestimmenden Steuerungsmechanismen des Verwaltungsrechts « ${ }^{47}$.

Je nach der Position am Markt zeigt der realisierte »schlanke Staat « ein unterschiedliches Antlitz: Die bunte Vielfalt der neuen Informations- und Kommunikationstechniken wird von vielen als Bereicherung ihrer persönlichen Entfaltungsmöglichkeiten erlebt, während andere wiederum die Schließung des altvertrauten Postamts im Stadtteil ${ }^{48}$ als Verlust wahrnehmen und der Verteuerung des Brief- und Paketbeförderung alternativlos ausgeliefert sind ${ }^{49}$. Es geht hierbei eben nicht nur um das jeweils unterschiedliche Know-how, also die Kompetenz zur Handhabung der neuen IuKTechniken, sondern auch um den allmählichen Abschied vom »Verwöhn-Aroma des Sozialstaats « ${ }^{5 \circ}$. Wenn die ehemals vom Staat erbrachten Infrastrukturleistungen und selbst die Gewährleistung persönlicher Sicherheit immer mehr zur Ware werden, verfestigt sich die »Struktur der sozialen Ungleichheit «" ${ }^{\text {" }}$. Das Projekt des »Schlanken Staates « zeigt seinen Januskopf:»Weniger Staat « zahlt sich aus - aber nur für manche.

\title{
Maren Witthoeft \\ Hans Litten - Ein zu Unrecht fast vergessener Anwalt der Opfer
}

Weiche Gesichtszüge, volle Lippen, eine hohe Stirn, die Haare bereits auffallend gelichtet, eine runde, von dicken Rändern gefaßte Brille, ein sensibler und zugleich kritischer Blick: der Berliner Rechtsanwalt und bekannte Strafverteidiger der Weimarer Republik Hans Achim Litten, jener Mann, der Hitler in die Enge trieb - und dafür mit seinem Leben bezahlen mußte. Das Konzentrationslager Dachau war die

VVDStRL 5s (1996), 7 (17). In der libidinösen Besetzung des Staates weniger eindeutig: J. Isensee, Staat und Verfassung, HdBStR I, ( 1987 ), \& I 3 Rdn. 3, I I; kritisch zum Etatismus A. Rinken, Einfuhrung in das juristische Studium, 2. Aufl. München 1991, S. 2 1 0 f.

46 Hierzu auch W. Hassemer, Aufgaben des Strafrechs in privater Hand? in: F. Sack u. a. (Fn. 37), 206 (216); W.-D. Narr (Fn. 38), S. i f

47 K. Stern, Postreform zwischen Privatisierung und Infrastrukturgewahrleistung. DVBl. 1997, 309 (312).

48 Bis zum Jahr 2000 soll das Netz von ehemals 16500 Postämtern auf etwa 10000 Filialen reduziert werden, FR v. 6. I1. 1996.

49 Von günstigeren Tarifen werde nur die Wirtschaft profitieren, meldete die FR am I0. 10. 1997. "Kleineren Gewerbetreibenden und anderen gemeinen Kunden drohen eher höhere Preise*.

50 D. Merten (Fn. 45), S. i 7; zum Stellenwert des Sozialstaatsprinzips in diesem Kontext vgl. M. Kutscha (Fn. 3), S. 396 f., zur fruheren Diskussion bereit ders., Vom zeitgemäßen Sozialstaatsverständnis, KJ I 982 , $3^{8} 3$.

51 T. v. Trotha (Fn. 37), S. 33. 
letzte Station auf dem fünfjährigen Leidensweg des jungen Litten durch die Gefängnisse und KZs seiner Häscher: Sonnenburg, Brandenburg, Esterwege, Lichtenburg, Buchenwald und schließlich Dachau. Hier nahm er sich, durch die fünf Jahre währenden systematischen Mißhandlungen in den Selbstmord getrieben, in der Nacht auf den 5. Februar 1938 - vor 60 Jahren -das Leben. Seine Mitgefangenen im sogenannten Judenblock, von denen Herbert Haarburger am 7. Februar dieses Jahres auf einer Gedenkveranstaltung zum 60. Todestag von Hans Litten in der KZ-Gedenkstätte Dachau anwesend war, fanden ihn am Morgen auf dem Abort. Er hatte sich erhängt. Hans Litten gehörte neben Rudolf Olden, Alfred Apfel und Max Hirschberger zu jenen Strafverteidigern der Weimarer Republik, die eine hohe Verteidigerkultur begründet haben. Damit machte sich Litten zwangsläufig zum Feind der aufstrebenden Nationalsozialisten, vor allem weil Litten in erster Linie deren Opfer vor Gericht vertrat.

\section{Ein rebellischer Geist}

Littens politische und berufliche Laufbahn hatte ihn früh zum Gegner der Reaktion gemacht. Bereits vor der Machtergreifung 1933 hatte ihn die Rechte im Visier, ohne Begleitschutz konnte der junge Anwalt das Haus nicht mehr verlassen. Nach dem Edenpalast-Prozeß 193I sah sich Litten immer häufiger Angriffen der nationalsozialistischen Presse ausgesetzt. Der »Angriff « etwa forderte: »Legt dem Anarchisten endlich das unsaubere Handwerk! « Zudem wurde er bei der Anwaltskammer denunziert, denn lange vor der Machtergreifung der Nazis gab es unter den Richtern und Staatsanwälten, aber auch in der Anwaltschaft die Tendenz, sich kritischer Verteidiger auf diese Weise zu entledigen.

Die politische Entwicklung des am 19. Juni 1903 in Halle geborenen Hans Achim Litten hat seinen Ursprung in der Jugendbewegung. Margot Fürst, politische Mitstreiterin Littens und von I 93 I bis 1933 Sekretärin in dessen Anwaltsbüro, berichtete auf der Gedenkveranstaltung in Dachau über ihre Zusammenarbeit und das Leben mit Litten. Nach Dachau hatten u.a. die Lagergemeinschaft Dachau, der Deutsche Anwaltsverein, der Republikanische Anwältinnen- und Anwälteverein und der Förderverein für Internationale Jugendbegegnung in Dachau geladen.

Mit Litten und ihrem Ehemann Max Fürst hatte Margot Fürst in der heutigen Berliner Zolastraße jene Wohnung geteilt, aus der Litten am 28. März 1933 im Morgengrauen verhaftet wurde. Die heute in Stuttgart lebende 8 jjährige Margot Fürst, Nachlaßverwalterin des Künstlers HAP Grießhaber, traf Litten und ihren späteren Mann Max - ein Jugendfreund Littens - zum ersten Mal 1927 auf einem Bundestag der Jugendbewegung bei Nürnberg. Alle drei gehörten dem "Schwarzen Haufen" an, einer Abspaltung des deutsch-jüdischen Wanderbundes, der zu Wandervogel und Jugendpflege auf Distanz ging und sozialrevolutionäre Ideen vertrat. "Haupttheoretiker des , Schwarzen Haufens` war Hans «, berichtet Margot Fürst, "er war Marxist, ohne je parteipolitisch organisiert gewesen zu sein. Der Marxismus war ihm nützliches Handwerkszeug, um gesellschaftliche Zusammenhänge zu analysieren «.

Litten hatte im Verlauf seiner Jugend eine feine Wahrnehmung für soziale Ungerechtigkeit und Ausgrenzung, für Doppelzüngigkeit und Opportunismus entwickelt. Er wuchs in einer großbürgerlichen, konservativ bis monarchisch gesinnten Königsberger Familie auf. Der Großvater war Vorsteher der jüdischen Gemeinde in Königsberg gewesen, die Mutter Irmgard Litten entstammte einer Pastoren- und Professorenfamilie und der Vater Fritz Litten war Dekan der juristischen Fakultät der Königsberger Universität und zeitweilig deren Rektor. Der Vater, dessen Haus zum 
Treffpunkt konservativer Gegner der Republik wurde, war aus Karrieregründen vom Judentum zum Christentum konvertiert, was Sohn Hans als opportunistischen Akt kritisierte und sich darauf hin umso intensiver mit religiösen Themen, vor allem dem Judentum, auseinandersetzte. In Opposition zum Vater festigte Hans schon in frühen Jahren seinen rebellischen Geist. Dies kam auch in Äußerlichkeiten, wie seiner langen Haartracht, zum Ausdruck - in Zeiten preußischen Kadavergehorsams eine Provokation.

\section{Anwalt der Opfer}

Litten nahm Mitte der zwanziger Jahre sein Studium der Jurisprudenz in Berlin und München auf, seinem Vater zu Liebe. Ein in der Zeit der Jugendbewegung entwikkeltes Berufsziel war es gewesen, Hafenarbeiter zu werden, was ihm jedoch seine Freunde Max und Margot ausreden konnten, mit der Begründung, er sei zu intelligent und könne als Anwalt mehr bewirken.

Littens Hauptinteresse und seine Begabung lag allerdings im kunstgeschichtlichen und musischen Bereich. Diese Vorliebe erlosch auch in der Zeit der Haft nicht. In einem Brief an seine Mutter aus dem KZ schreibt Litten: "Ich kann Dir Bach'sche Musik nur immer wieder ganz dringend empfehlen. Man wird von der Gewalt und Objektivität dieser Musik so herrlich ruhig, und alle Aufregungen werden unwichtig ... Ich bin jedesmal glücklich, wenn ich Sonntags im Leipziger Sender eine BachKantate ... hören kann. Das ist zur Zeit überhaupt so ziemlich das einzig Erfreuliche für mich.«

I 928 begann Litten seine anwaltliche Tätigkeit in Berlin. Einen leichten Stand hatte er nicht, die Justiz war geprägt von der Kontinuität der alten Eliten des Kaiserreichs, die der Republik überwiegend feindlich gesinnt waren und so den späteren Übergang ins Dritte Reich relativ bruchlos vollziehen konnten. Im beruflichen Umfeld Littens wuchs der Einfluß der Rechten, die Unparteilichkeit der Justiz geriet schleichend unter die Räder. »Unerklärlich war das schließlich nicht; die Beamten dachten an ihre Zukunft, und die Zukunft gehörte offenbar irgendeiner Art der nationalistischen Reaktion«, schreibt Littens Kollege Rudolf Olden.

Littens Zivilpraxis diente in erster Linie der Sicherung des Lebensunterhalts und der Fortführung der Praxis, die wenig lukrative Strafpraxis hingegen umfaßte den wesentlichen Teil seiner Arbeit. Die Strafprozesse, in denen er vornehmlich als Verteidiger angeklagter Kommunisten auftrat oder als Nebenkläger und Anwalt der Opfer, wenn Vergehen der Nationalsozialisten zur Anklage kamen, wurden in der Regel von der "Roten Hilfe«, der Rechtshilfeorganisation der KPD, finanziert. Dies verlief nicht ohne Konflikte, denn das Ziel der KPD deckte sich nicht unbedingt mit Littens Anliegen, stets das Individuum in den Mittelpunkt seiner Bemühungen um Gerechtigkeit zu stellen, nie Partei- oder Staatsinteressen. Der Partei hingegen war daran gelegen, Märtyrer im Kampf gegen Rechts zu schaffen. Rudolf Olden Journalist und Rechtsanwalt beim Berliner Kammergericht, u. a. Verteidiger Carl von Ossietzkys im berühmten "Soldaten sind Mörder «-Prozeß von 1931, der 1933 nach Oxford emigriert war und 1940 bei der Schiffsüberfahrt in die USA durch einen deutschen U-Bootangriff ums Leben kam - schreibt in seinem Vorwort zu Irmgard Littens 1940 erschienenen Erinnerungen »Eine Mutter kämpft gegen Hitler «' : „Übrigens war es nicht so lange nachher, daß seine Tätigkeit in politischen Prozessen abnahm; die Rote Hilfe... ließ ihm kein Mandat mehr zukommen. Warum? ... Litten war zu erfolgreich. Der kommunistischen Agitation war mit Bluturteilen und 
mit Märtyrern in den Zuchthäusern gedient, nicht mit Freisprüchen und mit gerechten Entscheidungen der bürgerlichen Gerichte.«

Litten investierte viel Energie und Zeit in eigene Recherchen, auch weil er die Staatsanwaltschaft und Richterschaft der Voreingenommenheit gegenüber seinen Mandanten und der Nähe zur nationalsozialistischen Bewegung verdächtigte. So überrascht es nicht, daß - wie Margot Fürst in Dachau berichtete - häufig bis zum Morgengrauen gearbeitet wurde. »Morgens begann ich um etwa $10.30 \mathrm{Uhr}$, abends gingen wir ins Café, um danach weiterzuarbeiten bis zwischen 3 und 6 Uhr. Auch am Sonntag wurde gearbeitet. Der Samstagnachmittag allein war Max' und meine Zeit." Rudolf Olden charakterisiert seinen Kollegen als einen vorzüglichen Kenner des Prozeßrechts und einen "gründlichen Verteidiger, der offenbar unbegrenzte Zeit und unbegrenzte Geduld hatte und auch Geringfügiges wichtig nahm, wenn es im Interesse seines Mandanten zu liegen schien «.

In kritischen Juristenkreisen ist Hans Litten durch seine damals Aufsehen erregende Prozeßführung kein Unbekannter geblieben. Bereits 1929 trat Litten mit einer Strafanzeige gegen den sozial demokratischen Polizeipräsidenten von Berlin, Zörgiebel, wegen Mordversuchs in 33 Fällen hervor. Anlaß war ein Verbot aller politischen Veranstaltungen unter freiem Himmel von 1928, das auch für den I. Mai 1929 nicht aufgehoben wurde, vielmehr mit der Anweisung bekräftigt wurde, auf Demonstranten, die dieses Verbot mißachten, zu schießen. Es gab zahlreiche Tote und über 100 Verletzte.

Littens Nebenklagetätigkeit im »Edenpalast«-Prozeß und seine Verteidigung im »Felsenecke«-Prozeß sind in die Justizgeschichte eingegangen. Im »Edenpalast «Prozeß von I93 I trat Litten als Nebenklagevertreter auf. Gegen Angehörige der SA wurde wegen Totschlags in drei Fällen, Landfriedensbruch und Körperverletzung ermittelt. Anlaß der Anklage war der gewaltsame Übergriff des »SA-Sturm 33《 auf eine Versammlung des Arbeiterwandervereins »Falke« im Berliner Tanzpalast »Eden«. Die Mitglieder des Vereins wurden mißhandelt, dann stürmte die SA den Tanzboden und schoß auf die Gäste. Litten hatte mit Erfolg die Ladung Hitlers als Zeuge vor das Kriminalgericht Berlin-Moabit beantragt. Er wollte durch die Befragung Hitlers nachweisen, daß die Parteiführung der NSDAP Gewalttaten nicht nur duldete, sondern vielmehr mit propagandistischen Mitteln förderte und plante und damit der wenige Monate vorher abgelegte »Legalitätseid « vor dem Reichsgericht eine Farce war. Es gelang dem damals gerade 27jährigen Litten durch präzise Quellenkenntnis und Verlesen von Zitaten aus parteioffiziellen Schriften der NSDAP, Hitler die legalistische Maske vom Gesicht zu reißen, jenem Mann, der nicht aufhörte zu betonen, so auch in der Gerichtsverhandlung am 8. Mai 293 I, daß »unsere SA das strengste Gebot (hat), sich von Angriffen gegen Andersdenkende fernzuhalten«. Hitler wurde von Litten in einer zweistündigen Befragung derart in die Enge getrieben, das dieser schließlich nach der Konfrontation mit Goebbels Schriften mit hochrotem Kopf in den Gerichtssaal schrie: »Wie kommen sie dazu, Herr Rechtsanwalt, zu sagen, es ist eine Aufforderung zur Illegalität? Das ist eine durch nichts zu beweisende Erklärung! « Daß Litten mit diesem juristischen Erfolg - die SA-Angehörigen wurden verurteilt - sein eigenes Todesurteil besiegelte, ahnte damals niemand.

Doch der »Edenpalast «-Prozeß war nicht der einzige Prozeß, in dem Litten den Kern der NS-Ideologie entlarvte und zur Verurteilung von SA-Mitgliedern beitrug. Es folgten bis 1933 fünf weitere Prozesse gegen Angehörige des "SA-Sturm 33«. Genannt seien hier der Röntgenstraßenprozeß, in dem neun Kommunisten angeklagt waren. Anlaß war eine Schießerei zwischen Kommunisten und Nationalsozialisten vor einem SA-Lokal, bei der ein SA-Mann getötet wurde. Litten konnte beweisen, das 
die nationalsozialistischen Zeugen nicht die Wahrheit gesagt hatten, die Täter waren

SA-Leute gewesen. Littens Mandanten wurden freigesprochen.

Im selben Jahr fand der »Felsenecke «-Prozeß statt, in dem wiederum ein Überfall der SA auf Kommunisten zur Verhandlung stand. Das Verfahren ist benannt nach dem Ort des Geschehens, der Arbeiterkolonie Felsenecke, in der Kommunisten und Sozialdemokraten wohnten, auf die i $5 \circ$ SA-Schläger einen Überfall verübt hatten, wobei ein Komunist und ein SA-Mann zu Tode kamen. Litten verteidigte einen Komunisten, dem der Mord an dem SA-Mann Schwarz zu Last gelegt wurde. Litten wurde als unbequemer Verteidiger nach monatelangen Verhandlungen vom Schwurgericht ausgeschlossen. Begründung: Er habe "eine hemmungslose parteipolitische Propaganda im Prozeß entfaltet, zahlreiche Anträge "nur aus politischem Sensationsbedürfnis" gestellt. Litten legte Beschwerde gegen den Beschluß ein und hatte Erfolg. Der Prozeß platzte. Schließlich bleib er aber auch unter neuem Vorsitz ausgeschlossen. Die Schuldigen wurden nie ermittel. Kaum waren die Nazis an der Macht, nahmen sie u.a. dieses Verfahren zum Anlaß, den kritischen Rechtsanwalt zu inhaftieren. Während des Wiederaufnahmeverfahrens wurde Litten schwer gefoltert, um ihn zur Denunziation seines Mandanten zu bewegen. Unter der Folter nannte er Namen, wiederrief aber in der selben Nacht. Litten überlebte nur knapp einen Selbstmordversuch, mit dem er sich den weiteren Folterungen und Erpressungen entziehen wollte.

\section{Haft und Folter}

Litten gehörte zu den ersten Opfern der gerade an die Macht gelangten NSDAP. Einen Tag nach dem Reichstagsbrand, am 28. Februar 1933, wurde Litten in sogenannte "Schutzhaft « genommen. Als Grundlage der willkürlichen Verhaftungswelle, der zahlreiche politisch Andersdenkende, Kommunisten, Sozialdemokraten und Gewerkschafter zum Opfer fielen, diente die am selben Tag von Reichspräsident Hindenburg unterzeichnete »Notverordnung zum Schutz von Volk und Staat«. Diese Verordnung setzte auf unbestimmte Zeit Grundrechte außer Kraft und ließ den neuen Machthabern freie Hand, ihre politischen Gegner ohne gerichtliche Kontrolle auszuschalten. Wie exzessiv von dieser "Notverordnung " Gebrauch gemacht wurde, belegt die parteioffizielle Zahl von 27000 "Schutzhäftlingen«, die im Juli 1933 in den Gefängnissen und wilden KZs der Nazis saßen.

In der Nacht zum 28. Februar wurde Litten zunächst in eine SA-Kaserne verschleppt, um am nächsten Tag ins Gefängnis Spandau gebracht zu werden. Den einzigen Besuch, den er empfangen durfte, war der seiner Sekretärin Margot Fürst. Im Lager Sonnenburg, in das Litten Anfang April 1933 verlegt wurde, war er Angehörigen jenes "SA-Sturm 33 « ausgeliefert, die er während seiner Anwaltstätigkeit vor Gericht wiederholt in Bedrängnis gebracht hatte. Sie nahmen in ihrer Funktion als "Hilfspolizisten« gnadenlos Rache. Neben Litten waren zu jener Zeit auch Erich Mühsam und Carl von Ossietzky in Sonnenburg inhaftiert und, wie Litten selbst, schweren Mißhandlungen ausgesetzt. Litten wurde nach Intervention Margot Fürsts nach Spandau zurückverlegt, wo er nach den bereits erwähnten Vernehmungen in Sachen "Felsenecke«-Prozeß einen Selbstmordversuch beging und in das Moabiter Krankenhaus eingeliefert wurde.

Die Tortur nahm ab Oktober 1933 ihre Fortsetzung im KZ Brandenburg. Anfang 1934 wurde Litten schließlich nach Esterwege verlegt, wo er unter der schweren Arbeit im Moor zu leiden hatte. Seine Mutter erstritt sich auch hier nach langem Kampf mit dem zuständigen Staatsanwalt der Gestapo eine Besuchserlaubnis. Sie fand Litten unter großen Schmerzen und im Fieber in der Krankenbaracke des 
Lagers. Litten kam drei Monate ins Krankenhaus, die Verletzungen konnten jedoch nie ganz ausheilen, ein Bein blieb auf Dauer steif. Im Juni 1934 wurde er ins $\mathrm{KZ}$ Lichtenburg verlegt, wo er über drei Jahre inhaftiert blieb. Er arbeitete in der Buchbinderei des Lagers und fand gelegentlich gar die Zeit, wissenschaftlich zu arbeiten, wobei sein Hauptinteresse der mittelalterlichen Literatur galt. Das Lager Lichtenburg wurde im September 1937 aufgelöst, die »Schutzhäftlinge « in das neu entstehende KZ Buchenwald verlegt. Buchenwald blieb für Litten jedoch nur eine Zwischenstation, im Oktober desselben Jahres erhielt die Mutter eine Karte ihres Sohnes: »Meine neue Adresse ist Dachau. Hans. « Die Unterschrift "Hans", statt Hans Achim, wie er sonst seine Briefe an die Mutter zeichnete, bedeutete: Auch hier bin ich den schweren Mißhandlungen der NS-Schergen unterworfen.

Die Nazis nahmen an einem ihrer meistgehaßten Feinde schonungslos Rache. Litten blieb auf Dauer in Haft, ohne daß je ein Strafverfahren gegen ihn eingeleitet worden wäre. Die Mutter Irmgard Litten wie auch der Bruder Heinz, Regisseur an der Berliner Volksbühne, unternahmen unzählige Versuche, Haftverschonung oder die Entlassung für Hans zu erreichen. Ihr unermüdlicher und mutiger Einsatz blieb trotz bester Verbindungen in etablierte gesellschaftliche Kreise ohne Erfolg. Sie mobilisierten Reichswehrminister von Blomberg, Reichsbischof Ludwig Müller, Wilhelm Furtwängler und sogar Freisler und Göring. Sie alle konnten - oder wollten - nichts bewirken. Sämtliche Gnadengesuche wurden zurückgewiesen. Hitler soll vor Zorn rot angelaufen sein, als er den Namen Litten hörte. Margot Fürst mobilisierte mittels Reisen nach Prag zu Max Brod, damals Redakteur am »Prager Tagblatt«, und in die Schweiz zu Hannah Arendt die Auslandspresse - ohne Erfolg. Auch der Befreiungsversuch, den Max und Margot Fürst für den 17. Dezember 1933 geplant hatten, schlug fehl. Sie waren Spitzeln der Gestapo aufgesessen. Sie wurden verhaftet. Max wurde zunächst im berüchtigten Columbiahaus und bis Februar 1934 in Oranienburg inhaftiert. Margot blieb bis September 1934 in Haft und erhielt im Zuge der "Hindenburgamnestie " Haftverschonung. Max und Margot Fürst verließen kurz darauf mit ihren beiden Kindern Deutschland in Richtung Israel.

Litten wurde systematisch in den Selbstmord getrieben. Er hielt den Schikanen nicht mehr stand. Körperlich und seelisch am Ende nahm Litten sich am 5. Februar 1938 das Leben. Bis kurz vor seinem Tod hatte er im Lager noch Vorträge über Literatur und Musik gehalten. Der ehemalige Mitgefangene Haarburger erinnert sich noch heute an Littens Ausführungen über die Musik Mozarts.

Irmgard und Fritz Litten emigrierten mit ihrem Sohn Heinz noch im Februar 1938 nach England.

\section{Ebrung für Hans Litten}

Cord Brüggmann, Mitglied des Dachauer Fördervereins und Jurastudent, sagte auf der Gedenkveranstaltung für Hans Litten, daß die Geschichte der Juristen in der Weimarer Republik »eher eine Tätergeschichte denn die eines Opfers wie Hans Litten" sei. "Spuren des Widerstandes gegen den Nationalsozialismus zu finden, ist fast unmöglich im Bereich Justiz oder Rechtswissenschaft. Viele gab es nicht, die uns als Vorbilder dienen könnten. « Die Justiz im Dritten Reich unterwarf sich unter Wahrung des Scheins von Rechtsstaatlichkeit - dem Vorbehalt der Politik. Die Richter und Staatsanwälte wurden zu willenlosen Gefolgsleuten der NS-Regierung. Als "ungeheuerliche Zumutung " hat Margot Fürst darum 1992 den Versuch der Berliner CDU-Fraktion bezeichnet, im Zuge der damaligen, teilweise über das Ziel hinausschießenden, Straßenumbenennungen auch den Namen Litten aus dem Stadtbild zu tilgen. Die im östlichen Berlin, am alten Justizpalast verlaufende Lit- 
tenstraße sollte auf Antrag der CDU umbenannt werden in "An der Klosterkirche«.

Margot Fürst schrieb in einem Brief an die damalige Berliner Senatorin für Justiz, Jutta Limbach: »Ich bin empört und entsetzt, daß es in der Bundesrepublik möglich sein soll, einem Kämpfer gegen Hitler und seine Bande schon vor I 933, der dafür von der Nacht des Reichstagsbrandes an bis zum Ende in Dachau am 5. Februar 1938 durch die KZs geschleift und mehrfach gefoltert wurde, die Ehrung zu bestreiten.« Die Bundesrepublik wie die Justiz im besonderen täte gut daran, sich eines Menschen wie Hans Litten in Würde zu erinnern und sich stark zu machen "für eine grundrechtsorientierte Justiz«, wie Jutta Limbach in ihrem Grußwort an die Gedenkveranstaltung formulierte.

Auf dem Gelände der KZ-Gedenkstätte Dachau verblieben 60 rote Rosen als Zeichen der Erinnerung an Litten und - wie es Max Mannheimer, ein Leidensgefährte Littens, in seinem Grußwort formulierte - als Zeichen der Hoffnung, daß die junge Generation die Leiden der Opfer des Nationalsozialismus im Gedächtnis behalte.

Redaktion Kritische Justiz (Hrsg.)

\section{Die juristische Aufarbeitung des Unrechts-Staats}

Stephan Beier · Susanne Benzler · Thomas Blanke - Gerd Denzel · Peter Derleder - Günter Frankenberg · Rüdiger Hachtmann · Heinrich Hannover - Ulrike Homann · Stefan Höpel · Herbert Jäger · Rolf Knieper · Helmut Kramer · Falko Kruse · Clea Laage - Manfred Messerschmidt - Ingo Mülller · Bettina Nehmer . Joachim Perels · Heidemarie Renk · Gerd Roellecke · Eberhard Rondholz · Hubert Rottleuthner · Christoph Schminck-Gustavus · Jürgen Seifert · Michael Stolleis · Margaretha Sudhof $\cdot$ Johannes Tuchel $\cdot$ Manfred Walther · Falco Werkentin · Manfred H. Wiegandt · Stefan Wittke · Hans Wrobel

Die Frage der Aufarbeitung von Staatsverbrechen stellte sich angesichts der Zerstörung des Rechts durch das NS-Regime in zuvor nicht gekanntem Umfang. Die Auseinandersetzung mit der - vom NS-System im Umfang und in der Struktur verschiedenen - Regierungskriminalität der DDR hat dem Thema eine neue Aktualität gegeben. Vor diesem Hintergrund untersuchen die Autoren des Bandes die rechtstechnische Funktionsweise des NS-Systems, die rechtstheoretische und strafrechtliche Auseinandersetzung mit der Diktatur des Nationalsozialismus nach 1945 sowie - in einem Ausblick - die justizielle Bewertung der SED-Herrschaft.

Die Repressionsmechanismen des NS-Regimes werden zunächst umfassend analysiert. Für die Zeit nach 1945 werden Kategorien für die Verdrängung bzw. Aufhellung des NS-Systems in der Rechtslehre ermittelt. Systematische Urteilsanalysen zeigen, wie vor allem mit dem kritiklosen Rückgriff auf das Normgefüge der NSDiktatur und der Überdehnung der Gehilfenkonstruktion bestimmte Gruppen von NS-Tätern ganz oder teilweise entlastet wurden.

Eine Untersuchung der anders verlaufenden Aufarbeitung der SED-Diktatur und der von ihr verordneten Repressionsakte rundet das Werk ab.

1998, 778 S., brosch., 89,-DM, 650,- $\ddot{O} S, 81,-s F r$, ISBN 3-7890-5054-7 